\title{
Current animal models of Alzheimer's disease: challenges in translational research
}

\author{
Mar Cuadrado-Tejedor ${ }^{1,2}{ }^{*}$ and Ana García-Osta ${ }^{1}$ \\ ${ }^{1}$ Neurobiology of Alzheimer's Disease, Neurosciences Division, Center for Applied Medical Research, CIMA, University of Navarra, Pamplona, Spain \\ 2 Department of Anatomy, University of Navarra, Pamplona, Spain \\ *Correspondence: mcuadrado@unav.es
}

Edited by:

Angel Cedazo-Minguez, Karolinska Institutet, Sweden

Reviewed by:

Marta Barrachina, Bellvitge Biomedical Research Institute (IDIBELL), Spain

Arjan Blokland, Maastricht University, Netherlands

Keywords: Alzheimer's disease, AD-mouse models, neuronal loss, multifactorial origin, therapeutics

\section{A commentary on}

Successful therapies for Alzheimer's disease: why so many in animal models and none in humans?

by Franco $R$, Cedazo-Minguez $A$. Front Pharmacol (2014) 5:146. doi: 10.3389/fphar.2014.00146

In their article, Franco and CedazoMinguez open the debate on why it is difficult to translate successful preclinical research in Alzheimer's disease (AD) mouse models into clinical practice (1). Here, we discuss some aspects that should be taken into account regarding the main discrepancies that exist between the current animal models and the disease in humans.

The translation of findings from bench to clinically relevant therapies is very complex. In fact, despite a full preclinical and clinical trial package, the large majority of drugs with initial phases based on translational-laboratory-based discoveries actually fail to complete the development process. A lack of efficacy, side-effects, inappropriate doses, and pharmacokinetics are just a few of the various reasons for this failure. Furthermore, the preclinical disease models on which new drugs are tested may not always be predictive of the effect of the agent in the human disease state (2). Could this be, as Franco and CedazoMinguez suggest, one of the major concerns in translational research in the case of $\mathrm{AD}$ ?

On the one hand, one of the main points to consider is probably the fact that most of the $\mathrm{AD}$-mouse models do not present the extensive neuronal loss observed in the brain of $\mathrm{AD}$ patients. At the moment of clinical diagnosis, most of the patients with AD-type dementia already have a Braak stage V or VI with a substantial synaptic and neuronal loss (3). Nevertheless, the loss of synapses is the best correlate of the cognitive impairment in patients with $\mathrm{AD}$ $(4,5)$. The synapse loss, which predates neuronal death in the human condition, is present in most of these mouse models, suggesting that they may represent the prodromal phase of the disease. Several authors have proposed that in the human condition, as a compensatory response, an enlargement of remaining synapses may occur, allowing the system to respond properly $(6,7)$. This could be one of the reasons why progression from early-phase to symptomatic stages in $\mathrm{AD}$ takes such a long time. It has been suggested that this "silent" period of the disease can even last for decades (8). Therefore, many of the therapies assayed on the AD models that are ineffective in people with the already established pathology might possibly be effective in preventing or delaying disease progression toward dementia. Although none of the animal models may represent the best option for evaluating novel therapeutic approaches for mild to moderate $\mathrm{AD}$ cases, they might be the first step in evaluating drugs that could reverse the synapse loss that underlies the "silent" phase of the disease. In animal models, the synapse loss underlies the memory deficits observed with the behavior tasks used for testing memory function. Therefore, therapeutic approaches for reversing memory deficits in $\mathrm{AD}$-mouse models through the enhancement of the synaptic function and/or spine density might be of great value for treating the memory decline that also occurs in patients with "mild cognitive impairment" (MCI), a term proposed by Petersen et al. as a new diagnostic entity for the transition between normal aging and AD dementia (9). Ultimately, since the $\mathrm{AD}$ drug development mainly motivated by the amyloid hypothesis had frightening results, the latest idea is that other pathways, which are not directly linked to $A \beta$, should be explored. In this context, phosphodiesterase-inhibitors, already on the market for other clinical uses (10) or epigenetic drugs (11) as potential memory enhancers could be a reliable option. Moreover, it is also important to note that all the $\mathrm{AD}$ therapies assayed in different clinical trials that could not continue on to subsequent phases due to the appearance of side-effects or those that have failed because the dose assayed in human trials had not been properly established, should also be carefully reviewed. Investing in the improvement of current drugs that have already been assayed and/or in drug-repurposing might be of special use in the case of $\mathrm{AD}$.

On the other hand, it should be taken into account that sporadic forms of $\mathrm{AD}$ have a multifactorial origin, with many different risk factors contributing to $\mathrm{AD}$ progression. Reducing any one of them by acting on/or improving the neural environment of the brain of these $\mathrm{AD}$-mouse models (by antioxidants, vitamins, cognitive enhancers, vasodilators, etc.) may be sufficient for ameliorating the incipient 


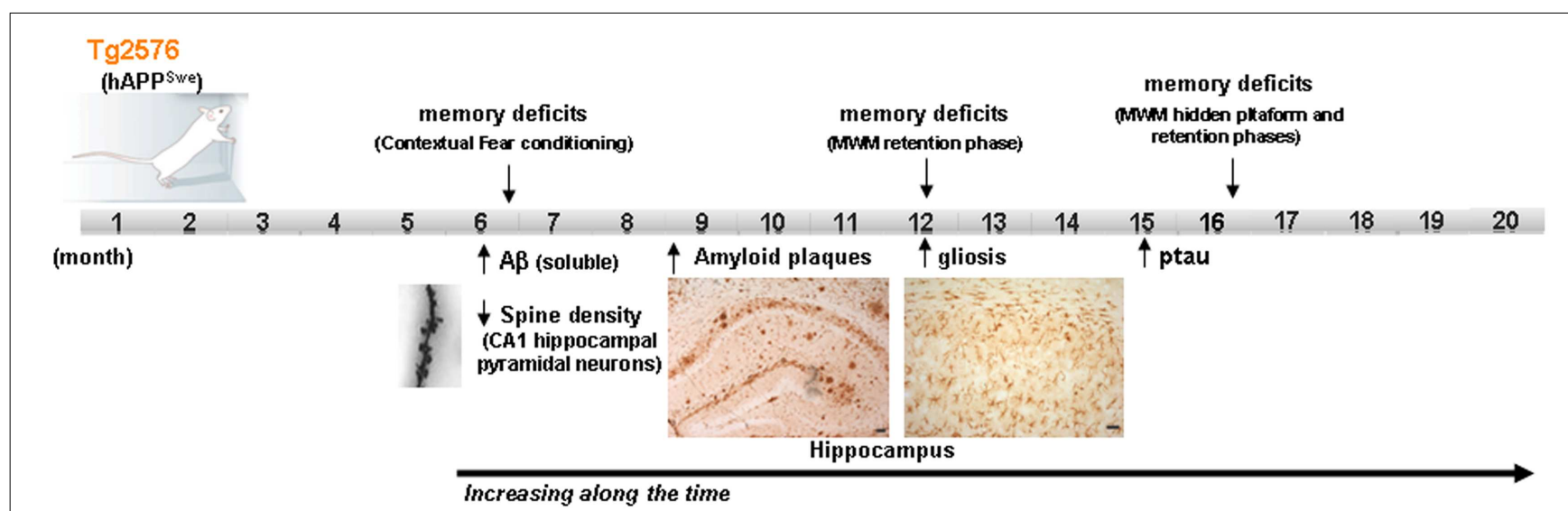

FIGURE 1 | Development of the different AD signs in Tg2576 mice over time. Scheme showing the time point of main AD features apparition in Tg2576 mice. At the age of 16 month, although no neuronal loss is presented in the brain of Tg2576 mice, the AD-phenotype is well established. MWM Morris water maze.

AD-phenotype of the models. Moreover, different mice strains should be used for modeling human-like environment factors because the use of inbred strains with a common genetic background, housed in a controlled environment, eliminates most of the variability that exists in the human condition. Some researchers have proposed that benefits with a new therapeutic intervention should be demonstrated in at least two different animal models and replicated by independent laboratories before beginning human experimentation (2). In addition, it is important to highlight that among the risk factors, aging, which is the most important one, is not always present in the preclinical studies carried out on animal models. The overexpression of familial AD-genes in these models accelerates the onset of the AD-phenotype, with amyloid plaques and synaptic deficits appearing even when the animals are $2-4$ months old. The possible advantage of using these early models, with their early onset of symptoms, has the disadvantage of compromising the age factor. Therefore, the use of late-plaque models (i.e., Tg2576, PDAPP, TgAPP23) for preclinical studies could be more accurate than using early plaque models (12). Figure $\mathbf{1}$ shows the main $\mathrm{AD}$ features developed in Tg2576 mice (a late-plaque model) over time; this model has been used in different studies in our laboratory over the last 10 years.
In summary, although we agree with most of the statements made in the review by Franco and Cedazo-Minguez, since animal models are indeed mandatory for preclinical studies, we consider that in the case of $\mathrm{AD}$, the model selected (a late onset model with an established phenotype) and the appropriate dosage regimen may be critical for the successful translation of experimental drugs to humans.

\section{REFERENCES}

1. Franco R, Cedazo-Minguez A. Successful therapies for Alzheimer's disease: why so many in animal models and none in humans? Front Pharmacol (2014) 5:146. doi:10.3389/fphar.2014.00146

2. Kieburtz K, Olanow CW. Translational experimental therapeutics: the translation of laboratorybased discovery into disease-related therapy. $M t$ Sinai J Med (2007) 74:7-14. doi:10.1002/msj. 20006

3. Serrano-Pozo A, Frosch MP, Masliah E, Hyman BT. Neuropathological alterations in Alzheimer disease. Cold Spring Harb Perspect Med (2011) 1(1):a006189. doi:10.1101/cshperspect.a006189

4. Terry RD. The pathogenesis of Alzheimer disease: an alternative to the amyloid hypothesis. J Neuropathol Exp Neurol (1996) 55(10):1023-5. doi:10.1097/00005072-199655100-00001

5. DeKosky ST, Scheff SW, Styren SD. Structural correlates of cognition in dementia: quantification and assessment of synapse change. Neurodegeneration (1996) 5:417-21. doi:10.1006/neur.1996.0056

6. DeKosky ST, Scheff SW. Synapse loss in frontal cortex biopsies in Alzheimer's disease: correlation with cognitive severity. Ann Neurol (1990) 27:457-64. doi:10.1002/ana.410270502

7. Scheff SW, Price DA. Synapse loss in the temporal lobe in Alzheimer's disease. Ann Neurol (1993) 33:190-9. doi:10.1002/ana.410330209
8. Selkoe DJ. Preventing Alzheimer's disease. Science (2012) 337:1488-92. doi:10.1126/science.1228541 9. Petersen RC, Smith GE, Waring SC, Ivnik RJ, Tangalos EG, Kokmen E. Mild cognitive impairment: clinical characterization and outcome. Arch Neurol (1999) 56:303-8. doi:10.1001/archneur.56.3.303

10. Garcia-Osta A, Cuadrado-Tejedor M, GarciaBarroso C, Oyarzabal J, Franco R. Phosphodiesterases as therapeutic targets for Alzheimer's disease. ACS Chem Neurosci (2013) 3:832-44. doi:10. $1021 / \mathrm{cn} 3000907$

11. Cuadrado-Tejedor M, Oyarzabal J, Pascual Lucas M, Franco R, Garcia-Osta A. Epigenetic drugs in Alzheimer's disease. Biomol Concepts (2013) 4(5):433-45. doi:10.1515/bmc-2013-0012

12. Lee JE, Han PL. An update of animal models of Alzheimer disease with a reevaluation of plaque depositions. Exp Neurobiol (2013) 22:84-95. doi: 10.5607/en.2013.22.2.84

Conflict of Interest Statement: The authors declare that the research was conducted in the absence of any commercial or financial relationships that could be construed as a potential conflict of interest.

Received: 17 July 2014; accepted: 08 September 2014; published online: 29 September 2014.

Citation: Cuadrado-Tejedor $M$ and García-Osta $A$ (2014) Current animal models of Alzheimer's disease: challenges in translational research. Front. Neurol. 5:182. doi: 10.3389/fneur.2014.00182

This article was submitted to Neuropharmacology, a section of the journal Frontiers in Neurology.

Copyright (c) 2014 Cuadrado-Tejedor and García-Osta. This is an open-access article distributed under the terms of the Creative Commons Attribution License (CC BY). The use, distribution or reproduction in other forums is permitted, provided the original author(s) or licensor are credited and that the original publication in this journal is cited, in accordance with accepted academic practice. No use, distribution or reproduction is permitted which does not comply with these terms. 